\title{
Die nächste Prionenerkrankung?
}

Fragestellung: Verhält sich fehlgefaltetes $\alpha$-Synuclein bei der Multisystematrophie (MSA) und dem idiopatischen ParkinsonSyndrom (iPS) wie infektiöses Prionprotein (z. B. bei der Creutzfeldt-Jakob-Erkrankung)?

Hintergrund: Beim IPS ist $\alpha$-Synuclein die Hauptkomponente der für die Erkrankung pathognomonischen intraneuronalen Lewy-Körperchen. Bei dem selteneren atypischen ParkinsonSyndrom MSA finden sich a-Synuclein-Ablagerungen im Zytoplasma von Gliazellen. Zunehmend weisen Ergebnisse aus der Grundlageforschung darauf hin, dass die toxischen Formen von a-Synuclein, die sich

Prusiner SB, Woerman AL, Mordes DA et al. Evidence for a-synuclein prions causing multiple system atrophy in humans with parkinsonism. Proc Natl Acad Sci U S A 2015;112: E5308-17 durch eine Fehlfaltung des Proteins auszeichnen, möglicherweise das nicht fehlgefaltete a-Synuclein modifizieren und sich somit im Gehirn wie Prionproteine verbreiten [1].
Patienten und Methodik: Prusiner et al. untersuchten diese Hypothese mithilfe von Gehirngewebshomogenaten von Menschen mit der klinischen und pathologischen Diagnose MSA $(n=14)$ und iPS $(n=6)$, die in HEK-Zellen und in den Thalamus von Mäusen injiziert wurden.

Ergebnisse: Alle Extrakte aus MSA-Gehirnen induzierten eine Aggregation von $\alpha$-synuclein im Zellmodell, während bei den Extrakten aus Parkinson-Gehirnen keine Aggregation beobachtet wurde. Ferner zeigten die mit MSA-Extrakten inokulierten transgenen Mäuse Koordinationsdefizite und histopathologisch neuronale Ablagerungen von $\alpha$-Synuclein, im Gegensatz zu den mit IPS-Extrakten inokulierten Tieren, die keine auffälligen Symptome oder derartige Neuropathologie aufwiesen.

Schlussfolgerungen: $\alpha$-Synuclein könnte im Sinne eines Prionproteins die Entstehung und Progression der MSA bedingen. Dieser Prozess scheint anders als die mögliche prionartige Ausbreitung anderer neurodegenerativer Erkrankungen zu sein.

\section{- Kommentar von Andrea Pilotto und Daniela Berg, Tübingen}

\section{Hypothese zur Ausbreitung der Neurodegeneration bei MSA}

In den letzten Jahren mehrt sich die Evidenz, dass die Verbreitung der typischen a-Synuclein-Ablagerungen bei Parkinson in ähnlicher Weise wie bei der klassischen Prionenerkrankung Creutzfeldt-Jakob Krankheit (CJD) abläuft: Fehlgefaltetes a-Synuclein „infiziert" normales a-Synuclein, sich ebenfalls fehl zu falten und in diesem Prozess zu der die Neurodegeneration induzierenden Toxizität beizutragen. Prusiner und Koautoren konnten nach Inokulation von MSA-Gehirnextrakten in HEK-Zellen und transgenen Mäusen Ablagerungen von phosphorylierten a-Synuclein nachweisen. Ferner zeigten die Tiere motorische Symptome. Bei Inokulation der Zellen und Mäuse mit Extrakten von IPS-Gehirnen konnten weder Ablagerungen nachgewiesen werden, noch fanden sich motorische Symptome. Dies untermauert die Hypothese, dass es sich bei der MSA um eine übertragbare neurodegenerative Erkrankung handelt.

Allerdings ergeben sich aus den Ergebnissen auch Fragen, die, um die Tragweite der Befunde einzuordnen, erwogen werden müssen. So konnten die Aggregate nur bei Menschen mit Mutationen nachgewiesen werden, die fehlgefaltetes Protein produzieren. Ohne eine derartige Mutation wurde keine Aggregation induziert. Die Tatsache, dass die hier verwendeten Tiere a-synuclein nur intraneuronal exprimieren, könnte Ursache hierfür sein - möglicherweise sind gliale Faktoren, wie sie bei den glialen Einschlüssen bei der MSA eine Rolle spielen, nötig, um auch nicht genetisch verändertes $a$-Synuclein zur aggregieren $[2,3]$. Ferner fanden sich in den Mäusegehirnen keine typischen neurodegenerativen Veränderungen, die bei der MSA im menschlichen Gehirn stark ausgeprägt sind. Dies könnte dadurch bedingt sein, dass ein Mausgehirn resistenter bezüglich
Proteinaggregation ist und nicht alt genug wird, um diese Veränderung aufweisen zu können. Hier muss berücksichtigt werden, dass auch die Ablagerung von A $\beta$-Plaques im AlzheimerMausmodell nicht zur Neurodegeneration führt [4], was darauf hinweisen könnte, dass die sich ausbreitenden Proteinablagerungen nicht die pathophysiologisch relevant toxischen sind, oder dass die Neurodegeneration ein multifaktorieller Prozess ist, und fehlgefaltetes Protein allein nicht ausreicht. Diese Fragen und auch die Frage nach der Wirkstärke von diesem möglichen Prionprotein für die Aggregation von a-Synuclein bei MSA müssen geklärt werden, bevor eine Übertragbarkeit diese wichtigen Befunde auf den Menschen möglich ist.
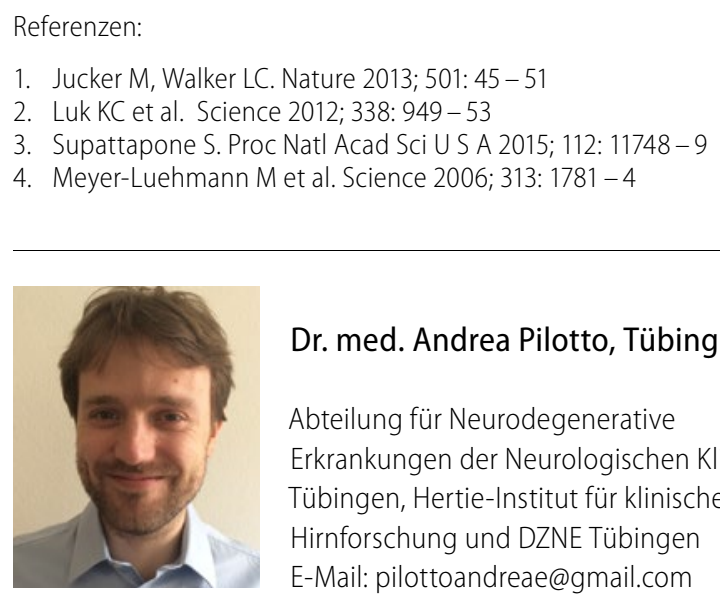

Dr. med. Andrea Pilotto, Tübingen

Abteilung für Neurodegenerative Erkrankungen der Neurologischen Klinik Tübingen, Hertie-Institut für klinische Hirnforschung und DZNE Tübingen E-Mail: pilottoandreae@gmail.com 